\title{
Perfil de contaminação por Staphylococcus e suas enterotoxinas e monitorização das condições de higiene em uma linha de produção de queijo de coalho
}

\author{
Contamination profile for staphylococci and its enterotoxins and monitorization of the conditions of \\ hygiene in a 'coalho' cheese production line
}

\author{
Maria de Fatima Borges ${ }^{I^{*}}$ Renata Tieko NassuI $^{\text {II }}$ José Luiz Pereira ${ }^{\text {III }}$ \\ Ana Paula Colares de Andrade ${ }^{\mathrm{IV}}$ Arnaldo Yoshiteru Kuaye ${ }^{\mathrm{V}}$
}

RESUMO

Avaliou-se o perfil de contaminação por Staphylococcus e suas enterotoxinas e monitorou-se as condições de higiene em uma linha de produção de queijo de coalho por meio da técnica de bioluminescência (ATP). A população de Staphylococcus $s p$. variou de $<1$ UFC $m L^{-1}$, no leite pasteurizado a $1,5 \times 10^{7} \mathrm{UFC} \mathrm{mL}^{-1}$ no leite cru, enquanto que a de Staphylococcus coagulase-positiva oscilou entre $<1 U F C \mathrm{~mL}^{-1}$ no leite pasteurizado e 5,0 x $10^{6} \mathrm{UFC} \mathrm{mL}^{-1}$ no leite cru. Staphylococcus coagulase-positiva foi detectada em 100\% (25/ 25) das amostras de leite cru e em 8\% (2/25) das amostras de queijos. Foram identificadas 12 espécies de Staphylococcus entre os 68 isolados selecionados, sendo nove coagulase negativa e três coagulase-positiva. No leite cru observouse alta freqüência de Staphylococcus coagulase positiva, principalmente $\boldsymbol{S}$. aureus, enquanto que em leite pasteurizado, coalhada e queijo verificou-se alta freqüência de Staphylococcus coagulase negativa e baixa freqüencia de Staphylococcus coagulase-positiva. A presença de enterotoxina estafilocócica foi constatada em $20 \%$ das amostras de leite cru e, conseqüentemente, também foi detectada no leite pasteurizado, na coalhada e no queijo produzido. A dosagem de ATP permitiu avaliar o estado de higienização das superfícies, o qual foi considerado: adequado em 62,1\% (36/95), “estado de alerta” em 23,2\% (22/95) e inadequado em 14,7\% (14/95) das superfícies. A detecção de espécies de Staphylococcus com potencial enterotoxigênico e a presença de enterotoxinas revelam a disseminação da contaminação na linha de produção do queijo de coalho provavelmente devido a falhas na aplicação das Boas Práticas de Fabricação desde a obtenção da matéria-prima até a produção final do queijo.

Palavras-chave: patógenos, intoxicação alimentar, produtos lácteos, Staphylococcus sp.

\begin{abstract}
This research aimed to evaluate the contamination by staphylococci and its enterotoxins as well as to monitor the conditions of hygiene from a coalho cheese production line, using ATP bioluminescence assay. Staphylococcus sp. population varied from $<1 C F U \mathrm{~mL}^{-1}$, in pasteurized milk to 1.5 $x 10^{7} \mathrm{CFU} \mathrm{mL}^{-1}$, in raw milk, whereas coagulase-positive staphylococci count ranged from $<1 C F U m L^{-1}$, in pasteurized milk to $5.0 \times 10^{6} \mathrm{CFU} \mathrm{mL}^{-1}$ in raw milk. Coagulase-positive staphylococci were detected in $100 \%(25 / 25)$ of the raw milk samples and in $8 \%(2 / 25)$ of cheese samples. Twelve Staphylococcus species were identified within the selected 68 isolates, being nine negative and three positive for coagulase. Raw milk samples showed a high rate of coagulase-positive, being $\mathbf{S}$. aureus the most common, whereas other product samples and equipment surfaces, pieces of furniture, utensils and manipulator gloves samples presented a high frequency of coagulase-negative and low frequency of coagulase-positive. Staphylococcal enterotoxin was detected in $20 \%$ of the raw milk samples and therefore in pasteurized milk, curd and cheese. ATP measurement permitted to assess the effectiveness of the surfaces cleaning, being considered adequate in 62.1\% (36/ 95), "alert state" in 23.2\% (22/95) and inadequate in $14.7 \%$ (14/95) of surfaces evaluated. Detection of staphylococci species with enterotoxigenic potential as well as enterotoxin presence reveal dissemination of contamination at the "coalho" cheese production line, possibly due inappropriate Good Manufacturing Practices (GMP) from the initial milking step until the final cheese production.
\end{abstract}

Key words: pathogen, food poisoning, dairy products, Staphylococcus sp.

IEmbrapa Agroindústria Tropical, CP 3761, 60511-110, Fortaleza, CE, Brasil. E-mail: fatima@cnpat.embrapa.br. Autor para correspondência.

"Embrapa Pecuária Sudeste, São Carlos, SP, Brasil.

IIIDepartamento de Ciências de Alimentos, Universidade Estadual de Campinas (Unicamp), Campinas, SP, Brasil.

${ }^{\text {IV }}$ Departamento de Tecnologia de Alimentos, Universidade Federal do Ceará (UFC), Fortaleza, CE, Brasil.

${ }^{\vee}$ Departamento de Tecnologia de Alimentos, Unicamp, Campinas, SP, Brasil. 


\section{INTRODUÇÃO}

A contaminação do queijo de coalho por Staphylococcus enterotoxigênicos coagulase-positiva e negativa representa um problema de saúde pública pelo risco de causar intoxicação alimentar. Essas bactérias, quando presentes em populações elevadas $\left(10^{5}-10^{6} \mathrm{UFC} \mathrm{mL}^{-1}\right.$ ou g $\left.^{-1}\right)$ e sob condições adequadas (temperatura, $\mathrm{pH}$, atividade de água e $\mathrm{O}_{2}$ ), produzem uma ou mais enterotoxinas estafilocócicas (SE) nos alimentos, as quais depois de ingeridas causam intoxicação.

A intoxicação estafilocócica é a causa mais freqüente de surtos de doenças microbianas transmitidas por alimentos, em muitos países. Surtos e casos esporádicos de intoxicação atribuídos ao consumo de produtos lácteos, principalmente queijos, têm sido relatados em vários países (CARMO et al., 2002; INPPAZ/OPS/OMS, 2006). No Brasil, os surtos investigados têm sido associados principalmente ao consumo de queijos do tipo Minas frescal, queijo Minas (CARMO et al., 2002) e de queijo de coalho (INPPAZ/ OPS/OMS, 2006).

Entre as espécies coagulase-positiva, $\boldsymbol{S}$. aureus é a mais freqüentemente associada a casos e surtos de intoxicação alimentar, devido à habilidade de muitas de suas cepas produzirem vários tipos de enterotoxinas (OMOE et al., 2005). Outras espécies produtoras de coagulase, como $S$. intermedius (BECKER et al., 2001) e $\boldsymbol{S}$. hyicus (ADESIYUN et al., 1984), também produzem enterotoxinas e têm sido envolvidas em alguns surtos. $\boldsymbol{S}$. intermedius podem ser potenciais causadores de intoxicação alimentar (KHAMBATY et al., 1994).

A produção de enterotoxinas por Staphylococcus coagulase-negativa como $S$. capitis, S. conhnii subsp cohnii, S. epidermidis, $S$. haemolyticus, S. hominis, S. saprophyticus, S. schleiferi, S. warneri, $S$. xylosus e $S$. chromogenes foi observada em vários estudos realizados sob condições de laboratório. Os resultados de alguns destes estudos sugerem que Staphylococcus coagulase-negativa (RODRIGUEZ et al., 1996; VERNOZY-ROZAND et al., 1996) pode ser causador de intoxicação alimentar em potencial. Já foram relatados alguns surtos de intoxicação estafilocócica associados a espécies coagulase-negativa (CARMO et al., 2002).

As enterotoxinas estafilocócicas são proteínas extracelulares de baixo peso molecular, hidrossolúveis e resistentes à ação de enzimas proteolíticas do sistema digestivo, permanecendo ativas após a ingestão. Outra característica importante é sua termoestabilidade, sendo capaz de resistir a tratamentos térmicos como a pasteurização e a ultrapasteurização. O número de enterotoxinas tem se expandido pela detecção de novos genes e, até o momento, já foram identificados 18 tipos distintos, mas com similaridade na estrutura e seqüência. As clássicas SEA, SEB, $\mathrm{SEC}_{1},{ }_{2}$, , SED e SEE são as de maior ocorrência. Outras treze enterotoxinas (SEG, SEH, SEI, SEJ, SEK, SEL, SEM, SEN, SEO, SEP, SEQ, SER E SEU) foram identificadas e seus genes (seg, seh, sei, sej, sek, sel, sem, sen, seo, sep, seq, ser e seu) correspondentes foram descritos (BLAIOTTA et al., 2004; OMOE et al., 2005).

A incidência de Staphylococcus coagulasepositiva, principalmente $\boldsymbol{S}$. aureus em queijo de coalho, é alta e tem sido relatada em vários estudos (BORGES et al., 2003; FEITOSA et al., 2003; LIMA, 2005). Na maioria desses estudos, os queijos são classificados como impróprios para o consumo humano, dada à constatação de níveis de contaminação superiores aos permitidos pela legislação (BRASIL, 2001). No Ceará, BORGES et al. (2003) avaliaram 43 amostras de queijo de coalho, produzido em 11 municípios pertencentes a cinco microrregiões produtoras de leite, e verificaram que $91 \%$ (39/43) das amostras estavam contaminadas por Staphylococcus coagulase-positiva em níveis superiores ao estabelecido pela legislação $\left(10^{3} \mathrm{UFC}^{-1}\right)$. Em outro estudo, FEITOSA et al., (2003) observaram que $72,7 \%$ (8/11) das amostras de queijo de coalho produzido em diferentes microrregiões do Rio Grande do Norte apresentavam contagens de Staphylococcus coagulase-positiva em níveis superiores ao estabelecido pela legislação.

A ocorrência de enterotoxinas estafilocócicas tem sido constatada com freqüência em produtos lácteos, especialmente em queijos, envolvidos ou não em surtos e casos esporádicos de intoxicação (CARMO et al., 1995; ROSEC et al., 1997; CARMO et al., 2002; NORMANNO et al., 2005).

A técnica imunoenzimática ELFA (Enzyme Linked Fluorescent Assay) permite a detecção simultânea de enterotoxinas estafilocócicas $\mathrm{A}, \mathrm{B}, \mathrm{C}_{1},{ }_{2},{ }_{3}$, D e E em fluidos sobrenadantes de culturas de Staphylococcus spp. e extratos de alimentos. Essa técnica tem sido empregada na pesquisa de enterotoxinas em queijos e na avaliação do potencial enterotoxigênico de Staphylococcus spp. isolados de alimentos (LIMA, 2005).

O presente estudo teve como objetivo avaliar a contaminação por Staphylococcus sp. e suas enterotoxinas na matéria-prima e no produto final e monitorar as condições de higiene das superfícies que contatam alimentos em uma linha de produção de queijo de coalho. 


\section{MATERIAL E MÉTODOS}

As amostras de alimentos e do ambiente foram coletadas em uma indústria de laticínios na região metropolitana de Fortaleza - CE, em intervalos de 4550 dias, no período de maio a outubro de 2004. Um total de 195 amostras foi coletado de cinco lotes de produção (I, II, III, IV e V), em 23 pontos da linha de processamento de queijo de coalho.

As contagens de Staphylococcus sp. e Staphylococcus coagulase-positiva foram realizadas em 100 amostras, sendo 25 de cada produto (leite cru, leite pasteurizado, coalhada e queijo), segundo metodologia do Bacteriological Analytical Manual/FDA(BENNETT \& LANCETTE, 2001). Para cada produto, foi calculado a média e o erro padrão das contagens. Após a incubação e a contagem das placas selecionadas, cinco a dez colônias características do gênero (sendo de uma a cinco típicas de $\boldsymbol{S}$. aureus) foram submetidas a testes bioquímicos (produção de catalase, coagulase e DNAse). A partir destes testes foram selecionados 68 isolados coagulase-positiva e negativa, para identificação em nível de espécie por meio do sistema de identificação de estafilococos e micrococos API-Staph (BioMérieux SA, Marcy-l'Etoile - France).

As 95 amostras de superfícies foram avaliadas por meio do método de detecção de ATP bioluminescência (Lightning $M V P^{\circledR}$ - BioControl Systems, Inc.). Neste sistema de monitoramento, o grau de higiene da superfície é classificado numa escala de zero a 7,5, em que a leitura de 0 a 2 indica superfície submetida à higiene rigorosa; $\leq 2,5$ superfície com higienização adequada; entre 2,6 e 3,0 indica alerta para a higienização da superfície e de 3,1 a 7,5 superfície com higienização inadequada (suja).

A pesquisa de enterotoxinas foi realizada em 20 amostras, sendo cada uma composta por cinco unidades amostrais dos produtos: leite cru, leite pasteurizado, coalhada e de queijo. Os extratos das amostras foram preparados de acordo com o protocolo do kit VIDAS ${ }^{\circledR}$ Staph enterotoxin II (BioMérieux SA, Marcy-l'Etoile, France) e analisados em um equipamento mini $V I D A S^{\circledR}$, que realiza automaticamente todas as etapas da reação até a leitura final. Resultado com leitura superior a 0,13 é considerado positivo para enterotoxinas estafilocócicas clássicas (A, B, $\mathrm{C}_{1},{ }_{2},{ }_{3}, \mathrm{D}$ eE).

\section{RESULTADOS E DISCUSSÃO}

O leite cru apresentou elevada população de Staphylococcus sp. e de Staphylococcus coagulase- positiva em $100 \%$ (25/25) das amostras. As contagens oscilaram entre $3,3 \times 10^{4}$ a $1,5 \times 10^{7} \mathrm{UFC} \mathrm{mL}^{-1}$, para Staphylococcus sp. e de 8,0 x $10^{3}$ a 5,0 x 10 $0^{6} \mathrm{UFC} \mathrm{mL}^{-1}$, para Staphylococcus c oagulase-positiva (Tabela 1). Este nível de contaminação, principalmente, por Staphylococcus coagulase positiva é considerado alto (superior a 1,0 x $10^{5} \mathrm{UFC} \mathrm{mL}^{-1}$ ) e pode favorecer a produção de enterotoxinas estafilocócica sob condições ambientais adequadas.

LAMAITA et al. (2005) também observaram alta freqüência Staphylococcus sp. (1,0 x $10^{5}$ a 2,5 x $10^{7} \mathrm{UFC} \mathrm{mL}^{-1}$ ) em leite cru estocado em tanques refrigerados de 80 propriedades rurais, em Minas Gerais.

No leite pasteurizado e na coalhada, foram observadas baixas contagens de Staphylococcus sp. e ausência de Staphylococcus coagulase-positiva (Tabela 1). No entanto, Staphylococcus coagulasepositiva, com identificação de $\boldsymbol{S}$. aureus, foi detectada nos queijos dos processamentos (lotes) III (2,0 x $\left.10^{2} \mathrm{UFC} \mathrm{g}^{-1}\right)$ e V $\left(1,9 \times 10^{2} \mathrm{UFC} \mathrm{g}^{-1}\right)$ (Tabela 1$)$. De acordo com a legislação (BRASIL, 1996), as contagens de Staphylococcus coagulase-positiva estavam acima dos limites estabelecidos $\left(1,0 \times 10^{2} \mathrm{UFC}^{-1}\right)$. A presença deste grupo de bactérias nos queijos indica que houve recontaminação após a pasteurização e, portanto, falhas na aplicação das Boas Práticas de Fabricação.

Em geral, os estudos sobre a qualidade microbiológica de queijo de coalho têm relatado índice de contaminação por Staphylococcus coagulasepositiva superior aos limites estabelecidos pela legislação (FEITOSA et al., 2003; BORGES, 2003). No Ceará, LIMA (2005) constatou que 98,7\% (79/80) dos queijos avaliados estavam contaminados com Staphylococcus sp. (6,0 x 10 a 8,9 x 107 $\left.\mathrm{UFC} \mathrm{g}^{-1}\right)$ e 53,7\% (43/80) com Staphylococcus coagulase-positiva (4,4 x $10^{4}$ a 5,9 x $\left.10^{7} \mathrm{UFC} \mathrm{g}^{-1}\right)$. Em outro estudo, BORGES et al. (2003) detectaram níveis de contaminação por Staphylococcus coagulase-positiva, entre 3,0 x $10^{4}$ a 2,0 x $10^{9} \mathrm{UFC} \mathrm{g}^{-1}$, em 91\% (39/43) das amostras de queijos. No Rio Grande do Norte, FEITOSA et al. (2003) observaram incidência de Staphylococcus coagulasepositiva em nível acima do que preconiza a legislação, em 72,7\% (8/11) dos queijos analisados. SENA (2000) também constatou elevado índice $(98,1 \%)$ de contaminação por esse grupo de bactéria nos queijos comercializados em Recife, PE. RAPINI et al. (2002) constataram elevada população de Staphylococcus sp. $\left(4,7 \times 10^{4}\right.$ a $\left.2,0 \times 10^{7} \mathrm{UFC} \mathrm{g}^{-1}\right)$ em $100 \%$ dos queijos comercializados nas praias de Salvador e Maceió. Em Aracaju, NASCIMENTO et al. (2005) detectaram Staphylococcus sp. em 94\% das amostras de queijo assado (tratamento térmico de $55^{\circ} \mathrm{C}$ ) comercializados em vários pontos da cidade. 
Tabela 1 - Valores médios e respectivos erros padrão da contagem de Staphylococcus em uma linha de produção de queijo de coalho.

\begin{tabular}{|c|c|c|c|c|}
\hline \multirow{3}{*}{ Lote } & \multicolumn{2}{|c|}{--------------------Leite-------------------- } & \multirow{3}{*}{ Coalhada } & \multirow{3}{*}{ Queijo } \\
\hline & & & & \\
\hline & Cru & Pasteurizado & & \\
\hline \multicolumn{5}{|c|}{ Staphylococcus sp. (UFC $\mathrm{mL}^{-1}$ ou g $^{-1}$ ) } \\
\hline I & $8,2 \times 10^{6}\left(0,33 \times 10^{6}\right)$ & $3,2 \times 10^{1}(0,0)$ & $8,6 \times 10^{2}\left(0,40 \times 10^{2}\right)$ & $1,7 \times 10^{3}\left(0,03 \times 10^{3}\right)$ \\
\hline II & $3,5 \times 10^{5}\left(0,23 \times 10^{5}\right)$ & $2,9 \times 10^{1}\left(0,29 \times 10^{1}\right)$ & $5,5 \times 10^{2}\left(0,26 \times 10^{2}\right)$ & $9,8 \times 10^{1}\left(0,26 \times 10^{1}\right)$ \\
\hline III & $1,5 \times 10^{7}\left(0,14 \times 10^{7}\right)$ & $<1,0(0,0)$ & $2,2 \times 10^{2}\left(0,21 \times 10^{2}\right)$ & $2,6 \times 10^{3}\left(0,32 \times 10^{3}\right)$ \\
\hline IV & $2,8 \times 10^{5}\left(0,21 \times 10^{5}\right)$ & $1,6 \times 10^{1}(0,0)$ & $1,0 \times 10^{2}(0,0)$ & $1,0 \times 10^{3}\left(0,54 \times 10^{3}\right)$ \\
\hline $\mathrm{V}$ & $3,3 \times 10^{4}\left(0,16 \times 10^{4}\right)$ & $<1,0(0,0)$ & $<10^{1}\left(0,40 \times 10^{1}\right)$ & $1,2 \times 10^{3}\left(0,08 \times 10^{3}\right)$ \\
\hline \multicolumn{5}{|c|}{ Staphylococcus coagulase-positiva (UFC $\mathrm{mL}^{-1} \mathrm{ou} \mathrm{g}^{-1}$ ) } \\
\hline I & $3,4 \times 10^{6}\left(0,48 \times 10^{6}\right)$ & $<1,0(0,0)$ & $<10^{1}(0,0)$ & $<10^{1}(0,0)$ \\
\hline II & $9,1 \times 10^{4}\left(1,07 \times 10^{4}\right)$ & $<1,0(0,0)$ & $<10^{1}(0,0)$ & $<10^{1}(0,0)$ \\
\hline III & $5,0 \times 10^{6}\left(1,13 \times 10^{6}\right)$ & $<1,0(0,0)$ & $<10^{1}(0,0)$ & $2,0 \times 10^{2}\left(1,98 \times 10^{2}\right)$ \\
\hline IV & $1,3 \times 10^{5}\left(0,13 \times 10^{5}\right)$ & $<1,0(0,0)$ & $<10^{1}(0,0)$ & $<10^{1}(0,0)$ \\
\hline $\mathrm{V}$ & $8,0 \times 10^{3}\left(0,65 \times 10^{3}\right)$ & $<1,0(0,0)$ & $<10^{1}(0,0)$ & $1,9 \times 10^{2}\left(1,18 \times 10^{2}\right)$ \\
\hline
\end{tabular}

O perfil de espécies de Staphylococcus identificadas na linha de produção do queijo de coalho foi bastante diversificado (Tabela 2). Entre os 68 isolados característicos de Staphylococcus sp. avaliados, foram identificadas 12 espécies, sendo três coagulase-positiva e nove coagulase-negativa.

Tabela 2 - Perfil de espécies de Staphylococcus isoladas na linha de produção de queijo de coalho.

\begin{tabular}{|c|c|c|c|c|c|}
\hline \multirow{2}{*}{ Amostra } & \multicolumn{5}{|c|}{-------------------------------------Lote / Espécie (nº cepas)------------------------------------ } \\
\hline & I & II & III & IV & $\mathrm{V}$ \\
\hline \multicolumn{6}{|c|}{ Produto } \\
\hline Leite cru & $\begin{array}{l}\text { S. aureus (2) } \\
\text { S. intermedius (2) }\end{array}$ & S. aureus (3) & $\begin{array}{l}\text { S. aureus (4) } \\
\text { S. haemolyticus (1) } \\
\text { S.xylosus (1) }\end{array}$ & $\begin{array}{l}\text { S. aureus (5) } \\
\text { S. caprae (1) } \\
\text { S. hyicus (2) }\end{array}$ & $\begin{array}{l}\text { S. aureus (2) } \\
\text { S. caprae (1) } \\
\text { S.xylosus (1) }\end{array}$ \\
\hline Leite pasteurizado & $\begin{array}{l}\text { S. cohnii cohnii (1) } \\
\text { S. lentus (2) }\end{array}$ & S. capitis (1) & ND & S. saprophyticus (1) & ND \\
\hline Coalhada & $\begin{array}{l}\text { S. saprophyticus (1) } \\
\text { S. xylosus (1) }\end{array}$ & S. hominis (2) & NI & $\begin{array}{l}\text { S. caprae (1) } \\
\text { S. epidermidis (1) }\end{array}$ & ND \\
\hline Queijo & S. xylosus (3) & $\begin{array}{l}\text { S. epidermidis (2) } \\
\text { S. xylosus (1) }\end{array}$ & $\begin{array}{l}\text { S. aureus (1) } \\
\text { S. epidermidis (1) } \\
\text { S. haemolyticus (1) }\end{array}$ & $\begin{array}{l}\text { S. cohnii cohnii (1) } \\
\text { S. epidermidis (3) }\end{array}$ & $\begin{array}{l}\text { S. aureus (2) } \\
\text { S. lentus (1) }\end{array}$ \\
\hline \multicolumn{6}{|c|}{ Equipamento e utensílio } \\
\hline Tanque de recepção & $\begin{array}{l}\text { S. lentus (1) } \\
\text { S. xylosus (1) }\end{array}$ & $\begin{array}{l}\text { S. cohnii cohnii (1) } \\
\text { S. epidermidis (1) }\end{array}$ & S. caprae (2) & S. caprae (2) & S.xylosus (1) \\
\hline $\begin{array}{l}\text { Tubulação do } \\
\text { pasteurizador }\end{array}$ & ND & NI & ND & S. saprophyticus (1) & ND \\
\hline Tanque de coagulação & ND & S. lentus (2) & ND & NI & ND \\
\hline Lira vertical & S. xylosus (2) & ND & ND & ND & ND \\
\hline Lira horizontal & S. xylosus (1) & ND & ND & ND & ND \\
\hline Mesa de enformagem & ND & $\begin{array}{l}\text { S. saprophyticus (1) } \\
\text { S. xylosus (1) }\end{array}$ & NI & S. epidermidis (1) & S. aureus (1) \\
\hline $\begin{array}{l}\text { Prateleira da câmara de } \\
\text { secagem }\end{array}$ & ND & NI & S. xylosus (1) & ND & ND \\
\hline $\begin{array}{l}\text { Prateleira da sala de } \\
\text { embalagem }\end{array}$ & ND & S. lentus (1) & S. epidermidis (2) & S. epidermidis (1) & ND \\
\hline $\begin{array}{l}\text { Prateleira da câmara de } \\
\text { estocagem }\end{array}$ & S. xylosus (1) & NI & ND & S. epidermidis (1) & ND \\
\hline
\end{tabular}

ND: não-detectado; NI: não-identificado.

Ciência Rural, v.38, n.5, ago, 2008. 
No leite cru foram identificadas seis espécies de Staphylococcus, sendo três coagulase-positiva* e três coagulase-negativa. Porém, observou-se alta freqüência de espécies coagulase-positiva, com prevalência de $\boldsymbol{S}$. aureus em $67 \%$ (16/24) dos isolados, S. intermedius em 8\% (2/24) e $\boldsymbol{S}$. hyicus em $8 \%(2 / 24)$. Apenas $17 \%$ (4/24) dos isolados eram espécies coagulase-negativa. Já no leite pasteurizado foram identificadas as espécies $\boldsymbol{S}$. capitis, $\boldsymbol{S}$. cohnii cohnii, $\boldsymbol{S}$. lentus e $\boldsymbol{S}$. saprophyticus e, na coalhada, foram identificadas as espécies $S$. caprae, $S$. epidermidis, $S$. hominis; S. saprophyticus e S. xylosus, todas coagulasenegativa. No queijo, entre os 16 isolados obtidos, foram identificadas seis espécies, com prevalência de $\boldsymbol{S}$. epidermidis em 37,5\% (6/16) dos isolados, $\boldsymbol{S}$. xylosus em $25 \%$ (4/16), $\boldsymbol{S}$. aureus em 18,8\% (3/16), $\boldsymbol{S}$. cohnii cohnii em 6,2\% (1/16), S. haemolyticus em 6,2\% (1/16) e S. lentus em 6,2\% (1/16). Houve alta freqüência (81,3\%) de espécies coagulase-negativa (5/6) e baixa freqüência $(18,7 \%)$ de espécies coagulase-positiva (1/6) nas amostras. A presença de $\mathbf{S}$. aureus foi constatada em $12 \%(3 / 25)$ dos queijos analisados.

Os resultados obtidos neste estudo, para o leite cru, foram similares aos observados por LAMAITA et al. (2005), que identificaram oito espécies de Staphylococcus, com alta freqüência de espécies coagulase-positiva, tais como $\boldsymbol{S}$. aureus $(34,6 \%), \boldsymbol{S}$. hyicus (22,7\%) e $\boldsymbol{S}$. intermedius (3,2\%) isoladas de leite cru refrigerado. Espécies coagulase-negativa ( $\boldsymbol{S}$. epidermidis, $\boldsymbol{S}$. sciuri e $\boldsymbol{S}$. cohnii) também foram identificadas, mas em menor freqüência (31\%). Em um estudo similar com queijo de leite de cabra, VERNOZYROZAND et al. (1996) isolaram 133 cepas de Staphylococcus coagulase-negativa e 18 cepas coagulase-positiva. Entre as cepas coagulase-negativa, foram identificadas 14 espécies, sendo três das quais predominantes: $\boldsymbol{S}$. simulans, $\boldsymbol{S}$. epidermidis e $\boldsymbol{S}$. xylosus. Todas as cepas coagulase-positiva pertenciam à espécie $\boldsymbol{S}$. aureus. DE LUCA et al. (1997) isolaram 313 cepas de Staphylococcus sp. de 135 amostras de diferentes tipos de queijos e as espécies de maior ocorrência foram $\boldsymbol{S}$. epidermidis (45,9\%), S. hominis (42,2\%), S. xylosus (44,4\%) e S. cohnii (37,8\%).

A prevalência de espécies de Staphylococcus coagulase-negativa, com potencial enterotoxigênico, como $S$. capitis, $S$. caprae, $S$. conhnii cohnii, S. epidermidis, S. haemolyticus, S. hominis, $S$. saprofphyticus e $\boldsymbol{S}$. xylosus, nas amostras da linha de produção de queijo de coalho, pode representar um risco em potencial de produção de enterotoxinas no produto final, sob condições adequadas de temperatura, $\mathrm{pH}$, disponibilidade de oxigênio, atividade de água e concentração de cloreto de sódio. Portanto, recomenda-se que a presença de espécies de Staphylococcus coagulase-negativa não sejam ignoradas em investigações de casos suspeitos de intoxicação estafilocócica, uma vez que este grupo de patógenos, estando presente no alimento, oferece risco de causar intoxicação ao consumidor.

OLIVEIRA (1999) avaliou a produção de enterotoxinas por 10 cepas de Staphylococcus coagulase-negativa com potencial enterotoxigênico, pertencentes às espécies $\boldsymbol{S}$. epidermidis (3), $\boldsymbol{S}$. chromogenes (1), S. hominis (1), S. hyicus (1), S. warneri (2) e $\boldsymbol{S}$. xylosus (2), crescidas em leite em pó desnatado e presunto cozido. No leite, o crescimento das cepas inoculadas foi progressivo e alcançou níveis entre $10^{6} \mathrm{e}$ $10^{7} \mathrm{UFC} \mathrm{mL}^{-1}$. Das dez cepas estudadas, apenas $\boldsymbol{S}$. chromogenes e $\boldsymbol{S}$. warneri de origem bovina foram capazes de produzir enterotoxinas nos alimentos.

A higienização das superfícies que contam com o alimento na linha de produção foi adequada $(\leq 2,5)$ em $62,1 \%$ (36/95) das amostras avaliadas, alerta para melhoria nos procedimentos em 23,2\% (22/95) e inadequada em 14,7\% (14/95). Em todos processamentos, o estado de higienização da superfície das luvas utilizadas pelos manipuladores foi adequado e a superfície interna de $100 \%$ (5/5) das embalagens de polietileno apresentou estado de higiene rigoroso (Tabela 3). Em relação às superfícies dos móveis, equipamentos e utensílios, nenhuma apresentou estado de higienização adequado em todos processamentos (Tabela 3). Nas superfícies do tanque de recepção de leite cru, da mangueira para drenagem do soro, da mesa de enformagem, das prateleiras da câmara de maturação, da sala de embalagem e da câmara de estocagem, o estado de higienização foi inadequado $(\geq 2,6)$ entre um a quatro processamentos. Os resultados indicam que as condições de higiene dos móveis, equipamentos e utensílios são críticas. As prateleiras da câmara de maturação e da sala de embalagem eram de fibra de vidro (FIBRAV, Lambari MG) e a da câmara de estocagem, de madeira, materiais estes de difícil limpeza, principalmente, quando se trata de resíduos de proteínas e gorduras.

A presença de enterotoxina foi constatada em 20\% (4/20) das amostras analisadas. Todas amostras contaminadas (4) eram oriundas do processamento III, no qual enterotoxinas foram detectadas no leite cru e, conseqüentemente, também detectadas no leite pasteurizado, na coalhada e no queijo obtido a partir desse leite.

A ocorrência de enterotoxina na linha de produção de queijo de coalho pode ser atribuída à elevada população de Staphylococcus coagulasepositiva $\left(5,0 \times 10^{6} \mathrm{UFC} \mathrm{mL}^{-1}\right)$ detectada no leite cru 
Tabela 3 - Monitorização das condições de higiene de superfícies que contatam alimentos em uma linha de produção de queijo de coalho, pelo método ATP (Bioluminescência).

\begin{tabular}{|c|c|c|c|c|c|}
\hline \multirow{2}{*}{ Superfícies } & \multicolumn{5}{|c|}{--------------------------Lote / Grau de higienização*-------------------------- } \\
\hline & I & II & III & IV & $\mathrm{V}$ \\
\hline \multicolumn{6}{|c|}{ Equipamento } \\
\hline Tanque de recepção de leite & 2,7 & 1,8 & 2,7 & 3,1 & 3,8 \\
\hline Tanque de coagulação & 2,7 & 2,6 & 1,5 & 2,1 & 2,4 \\
\hline Tubulação de saída do pasteurizador & 2,5 & 2,5 & 2,7 & 2,8 & 2,5 \\
\hline \multicolumn{6}{|c|}{ Utensílio } \\
\hline Cilindro de retenção da coalhada & 1,7 & 1,7 & 2,3 & 2,4 & 2,7 \\
\hline Dessorador de tecido & 2,6 & 2,5 & 2,2 & 2,5 & 2,7 \\
\hline Forma & 2,6 & 2,2 & 1,7 & 2,4 & 2,6 \\
\hline Garfo & 1,8 & 1,6 & 1,7 & 2,3 & 2,7 \\
\hline Homogeneizador do leite & 1,2 & 1,5 & 1,9 & 2,2 & 2,7 \\
\hline Lira de corte horizontal & 2,3 & 2,2 & 1,7 & 2,4 & 2,9 \\
\hline Lira de corte vertical & 1,8 & 1,8 & 1,6 & 2,3 & 2,7 \\
\hline Mangueira para drenagem do soro & 3,8 & 2,7 & 1,6 & 2,5 & 2,5 \\
\hline \multicolumn{6}{|c|}{ Móvel } \\
\hline Mesa de enformagem & 2,4 & 2,6 & 2,8 & 2,4 & 4,2 \\
\hline Prateleira da sala de embalagem & 3,7 & 2,9 & 3,6 & 3,0 & 5,1 \\
\hline Prateleira da câmara de estocagem & 3,1 & 2,4 & 3,2 & 4,7 & 4,1 \\
\hline Prateleira da câmara de maturação & 2,9 & 3,5 & 2,9 & 3,5 & 4,1 \\
\hline \multicolumn{6}{|c|}{ Embalagem } \\
\hline Superfície interna da embalagem & 1,9 & 1,9 & 1,6 & 1,7 & 2,0 \\
\hline \multicolumn{6}{|c|}{ Luva } \\
\hline Luvas 1 & 2.2 & 2,5 & 2,0 & 2,4 & 2,5 \\
\hline Luvas 2 & 2,5 & 2,4 & 2,4 & 2,3 & 2,5 \\
\hline Luvas 3 & 2,0 & 1,7 & 2,2 & 2,3 & 2,4 \\
\hline
\end{tabular}

* Classificação do grau de higienização: 0 a $2 \rightarrow$ Superfície submetida à limpeza rigorosa; $=2,5 \rightarrow$ Superfície com higienização adequada; 2,6 a 3,0 $\rightarrow$ Alerta para a higienização da superfície; 3,1 a 7,5 $\rightarrow$ Superfície com higienização inadequada (suja).

(Tabela 1), associada à ocorrência de espécies de Staphylococcus enterotoxigênicos coagulase-positiva e negativa, tais como $\boldsymbol{S}$. aureus, $\boldsymbol{S}$. haemolyticus e $\boldsymbol{S}$. xylosus (Tabela 2). Este resultado era esperado, uma vez que a pasteurização não inativa as enterotoxinas estafilocócicas devido à termorresistência.

A ocorrência de enterotoxinas estafilocócicas em produtos lácteos parece estar mais relacionada à habilidade das cepas em produzir enterotoxinas do que ao grau de contaminação por Staphylococcus. RAPINI et al. (2002) evidenciaram a produção de enterotoxina (SEB e SEC) com contagens de Staphylococcus sp. em torno de $10^{4} \mathrm{UFC}^{-1}$. em queijo de coalho comercializado nas praias do Nordeste do Brasil. No entanto, a presença de enterotoxinas não foi detectada em 43 amostras de queijo de coalho com elevada população $\left(4,4 \times 10^{4}\right.$ a 5,9 x $\left.10^{7} \mathrm{UFC} \mathrm{g}^{-1}\right)$ de Staphylococcus produtores de coagulase, produzido no Ceará (LIMA, 2005). Em outro estudo, a presença de enterotoxinas também não foi detectada em queijos artesanais da região do Serro, em Minas Gerais, com contagens de $1,0 \times 10^{7} \mathrm{UFC} \mathrm{g}^{-1}$ de $\boldsymbol{S}$. aureus (PINTO et al., 2004).

\section{CONCLUSÕES}

O grau de contaminação do leite cru por Staphylococcus enterotoxigênicos, principalmente $\boldsymbol{S}$. aureus e $\boldsymbol{S}$. intermedius, se mostrou elevado o suficiente para a produção de enterotoxinas estafilocócicas no produto.

A constatação da presença de enterotoxinas clássicas (A, B, C, D e E) nas amostras do processamento III indica que as enterotoxinas estafilocócicas, uma vez presentes em leite cru, não são inativadas pelo processo de pasteurização e permanecem no leite pasteurizado, na coalhada e, conseqüentemente, no queijo, tornando o mesmo um risco à saúde do consumidor. Além disso, indica contaminação na linha de produção do queijo de coalho, possivelmente devido a falhas na aplicação das Boas Práticas de Fabricação desde a obtenção do leite até o produto final (queijo).

Ciência Rural, v.38, n.5, ago, 2008. 


\section{AGRADECIMENTOS}

Ao Conselho Nacional de Desenvolvimento Científico e Tecnológico (CNPq) (Processo nํ502929/20030) pelo suporte financeiro. À Embrapa Agroindústria Tropical e à Embrapa Gado de Leite pelo apoio técnico durante a execução da pesquisa.

\section{REFERÊNCIAS}

ADESIYUN, A.A. et al. Productions of enterotoxins by Staphylococcus hyicus. Veterinary Microbiology, Shannon, v.9, n.5, p.487-495, 1984.

BECKER, K. et al. Enterotoxigenic potential of Staphylococcus intermedius. Applied and Environmental Microbiology, Washington, v.67, n.12, p.5551-5557, 2001.

BENNETT, R.W.; LANCETTE, G.A. Staphylococcus aureus. In: FOOD DRUG ADMINISTRATION. (Ed.). Bacteriological analytical manual. 8.ed. Gaithersburg: FDA, 2001. Cap.12, p.12.1-12.5. Capturado em 20 abr. 2007. Online. Disponível na Internet http://www.cfsan.fda.gov/ ebam/bam-12.html.

BLAIOTTA, G. et al. PCR detection of staphylococcal enterotoxin genes in Staphylococcus spp. Strains isolated from meat and dairy products. Evidence for new variants of seG and seL in $\boldsymbol{S}$. aureus AB-8802. Journal of Applied Microbiology, Belfast, v.97, n.5, p.719-730, 2004.

BORGES, M.F. et al. Microrganismos patogênicos e em queijo de coalho produzido no Ceará, Brasil. Boletim do Centro de Pesquisa e Processamento de Alimentos, Curitiba, v.21, n.1, p.31-40, 2003

BRASIL. Ministério da Agricultura, Pecuária e Abastecimento. Portaria $n^{\circ} 146$ de 07 de março de 1996. Aprova os Regulamentos Técnicos de Identidade e Qualidade dos Produtos Lácteos. Capturado em 15 mar. 2007. Online. Disponível na Internet http://extranet.agricultura.gov.br/sislegisconsulta/consultarLegislacao.do.

BRASIL. Ministério da Saúde. Secretaria Nacional de Vigilância Sanitária. Resolução RDC n 12, de 02 de janeiro de 2001. Aprova regulamento técnico sobre os padrões microbiológicos para alimentos. Brasília, DF, 10 jan. 2001b. Capturado em 01 abr. 2007. Online. Disponível na Internet http://www.anvisa.gov.br/legis/resol/12-01rda.htm.

CARMO, L.S. et al. Staphylococcal food poisoning in Minas Gerais State (Brazil). Arquivos Brasileiros de Medicina Veterinária, Belo Horizonte, v.47, n.2, p.113-122, 1995.

CARMO, L.S. et al. Food poisoning due to enterotoxigenic strains of Staphylococcus present in Minas cheese and raw milk in Brazil. Food Microbiology, London, v.19, n.1, p.914, 2002.

DE LUCA, G. et al. Staphylococcus aureus in dairy products in the Bologna area. International Journal of Food Microbiology, Amsterdam, v.35, n.3, p.267-270, 1997.
FEITOSA, T. et al. Pesquisa de Salmonella sp., Listeria sp. e microrganismos indicadores higiênico-sanitários em queijos produzidos no estado do Rio Grande do Norte. Ciência e Tecnologia de Alimentos, Campinas, v.23, n.3, p.162-165, 2003.

INSTITUTO PANAMERICANO DE PROTECCIÓN DE LOS ALIMENTOS Y ZOONOSIS (INPPAZ) / OGANIZACIÓN PANAMERICANA DE LA SALUD (OPS) / OGANIZACIÓN MUNDIAL DELA SALUDE (OMS). Vigilancia epidemiológica. Sistema de información regional para la vigilancia epidemiológica de las enfermedades transmitidas por alimentos (SIRVETA). Capturado em 28 mar. 2006. Online. Disponível na Internet http://www.panalimentos.org/sirveta/e/salida2.asp.

KHAMBATY, F.M. et al. Application of pulse field gel electrophoresis to the epidemiological characterization of Staphylococcus intermedius implicated in a food-related outbreak. Epidemiology and Infection, London, v.113, n.1, p.75-81, 1994.

LAMAITA, H.C. et al. Contagem de Staphylococcus sp. e detecção de enterotoxinas estafilocócicas e toxina da síndrome do choque tóxico em amostras de leite cru refrigerado. Arquivos Brasileiros de Medicina Veterinária de Zootecnia, Belo Horizonte, v.57, n.5, p.702-709, 2005.

LIMA, A.F. Staphylococcus coagulase-positiva e enterotoxinas em queijo de coalho. 2005. 86f. Dissertação (Mestrado em Tecnologia de Alimentos) - Faculdade Engenharia de Alimentos, Universidade Federal do Ceará

NASCIMENTO, I.R. et al. Qualidade microbiológica do queijo de coalho submetido a tratamento térmico comercializado em Aracajú - SE. Revista do Instituto de Laticínios Cândido Tostes, Juiz de Fora, v.345, n.60, p.253-255, 2005.

NORMANNO, G. et al. Coagulase-positive Staphylococci and Staphylococcus aureus in food products marketed in Italy. International Journal of Food Microbiology, Amsterdam, v.98, n.1, p.73-79, 2005.

OLIVEIRA, A.M. Investigação do comportamento de estafilococos enterotoxigênicos coagulase-negativos, em alimentos. 1999. 102f. Tese (Doutorado em Ciências de Alimentos) - Departamento de Ciências de Alimentos, Universidade Estadual de Campinas.

OMOE, K. et al. Comprehensive analysis of classical and newly described staphylococcal superantigenic toxin genes in Staphylococcus aureus isolates. FEMS Microbiology Letters, Amsterdam, v.246, n.2, p.191-198, 2005.

PINTO, M.S. et al. Diagnóstico sócio-econômico e cultural dos produtores e avaliação microbiológica do queijo minas artesanal da região do Serro - MG. Revista do Instituto de laticínios Cândido Tostes, Juiz de Fora, v.59, n.339, p.86-92, 2004.

RAPINI, L.S. et al. Pesquisa de Salmonella sp., Escherichia coli, Listeria sp. e Staphylococcus sp. e detecção de 
enterotoxinas estafilocócicas em queijo tipo coalho. Revista do Instituto de laticínios Cândido Tostes, Juiz de Fora, v.57, n.327, p.60-65, 2002.

RODRIGUEZ, L. et al. Gram-positive, catalase cocci from dry cured Iberian ham and their enterotoxigenic potential. Applied and Environmental Microbiology, Washington, v.62, n.6, p.1897-1902, 1996

ROSEC, J.P. et al. Enterotoxin production by staphylococci isolated from foods in France. International Journal of Food Microbiology, Amsterdam, v.35, n.3, p.213-221, 1997.
SENA, M.J. Perfil epidemiológico, resistência a antibióticos e aos conservantes nisina e sistema lactoperoxidase de Staphylococcus sp. isolados de queijos de coalho comercializados em Recife-PE. 2000. $75 f$. Tese (Doutorado em Veterinária) - Faculdade de Veterinária, Universidade Federal de Minas Gerais.

VERNOZY-ROZAND, C. et al. Enterotoxin production by coagulase-negative staphylococcal isolated from goats and cheese. International Journal of Food Microbiology, Amsterdam, v.30, n.3, p.271-280, 1996. 